\title{
Extension of the Jiles-Atherton Model for Modelling the Frequency Dependence of Magnetic Characteristics of Amorphous Alloy Cores for Inductive Components of Electronic Devices
}

\author{
R. SZEWCZYK* AND P. FRYDRYCH \\ Industrial Research Institute for Automation and Measurements \\ Al. Jerozolimskie 202, 02-486 Warszawa, Poland
}

\begin{abstract}
The paper presents the results of modelling the influence of magnetizing field frequency on the magnetic characteristics of $\mathrm{Fe}_{40} \mathrm{Ni}_{38} \mathrm{Mo}_{4} \mathrm{~B}_{18}$ amorphous alloy in as-quenched state. An extension for the Jiles-Atherton model was applied and changes of the parameter $k$ during the magnetization process were considered. On the base of the experiment, the parameters of the Jiles-Atherton model were calculated for experimental hysteresis loop. Evolutionary strategies, together with the gradient optimisation method, were used. Moreover, the changes of parameters $c$ and $a$ as the function of frequency of magnetizing field were also taken into consideration. Finally, the high conformity between the experimental and modelling results was achieved. This high conformity indicates that the results both create new possibilities in modelling of properties of inductive components based on the amorphous alloys, as well as extend the possibility of quantitative description of magnetization process.
\end{abstract}

PACS numbers: 75.50.Kj, 75.60.Ej, 75.78.-n

\section{Introduction}

Physical models of magnetization process are mainly focused on the modelling of the quasi-static hysteresis loops [1], which are the most important from the theoretical point of view. On the other hand, technical applications of magnetic cores as inductive cores in electronic devices, such as fluxgate sensors [2], magnetoelastic sensors [3] or inductive components of switching mode power supplies [4], require the model of dynamic characteristics of the core during magnetization process. Inductive components are very often magnetized with unsymmetrical or transient wave forms (especially in switching mode power supplies), which additionally complicates the modelling of the magnetic characteristics, and electrical response of the component.

Among the most popular models of magnetization process [1], only the energy-based Jiles-Atherton ( $\mathrm{J}-\mathrm{A}$ ) model gives the possibility of modelling of the dynamic, magnetic characteristics of inductive core components made of anisotropic magnetic materials, e.g. amorphous alloys. Moreover, this model creates the possibility of modelling of mechatronic properties of the core [5], including influence of the temperature or mechanical stresses $[6,7]$. On the other hand, the accuracy of the results of application of the original $\mathrm{J}-\mathrm{A}$ model is not sufficient, from the technical point of view. In many cases, results of the modelling are only in a qualitative agree-

* corresponding author; e-mail: rszewczyk@onet.pl ment with the experimental results (e.g. modelling of the frequency dependences [8] of magnetic characteristics of Permalloy 80), whereas significant quantitative divergences between experimental results and the model were observed. These limitations of the original J-A model indicate the necessity for its development and experimental verification, especially for anisotropic magnetic materials, such as iron-nickel based amorphous alloys.

\section{The $\mathbf{J}-\mathbf{A}$ model and the concept of its extension}

In the J-A model for calculation of the anhysteretic magnetization $M_{\text {an }}$ in the real material, weighted sum of the anisotropic anhysteretic magnetization $M_{\text {aniso }}[6]$ and the isotropic anhysteretic magnetization $M_{\text {iso }}$ has to be considered [9]:

$$
M_{\mathrm{an}}=t M_{\text {aniso }}+(1-t) M_{\mathrm{iso}}
$$

where $t$ describes the participation of the anisotropic phase in the material. Presented in the literature $[6,9]$ equations for both anhysteretic magnetizations $M_{\text {iso }}$ and $M_{\text {aniso }}$ covers parameter $a$, which quantifies the domain walls density and parameter $\alpha$ which is representing inter domain coupling.

In the $\mathrm{J}-\mathrm{A}$ model the irreversible magnetization $M_{\text {irr }}$ can be calculated from the following differential Eq. [6]:

$$
\frac{\mathrm{d} M_{\mathrm{irr}}}{\mathrm{d} H_{\mathrm{eff}}}=\delta_{M} \frac{M_{\mathrm{an}}-M_{\mathrm{irr}}-W}{\delta k},
$$

where parameter $\delta$ depends on the first derivative of the effective magnetizing field $H_{\text {eff }}$, whereas parameter $\delta_{M}$ guarantees the avoidance of unphysical stages of the $\mathrm{J}-\mathrm{A}$ 
model for minor loops, in which the incremental susceptibility becomes negative [10]. The frequency dependence of magnetic characteristics in the $\mathrm{J}-\mathrm{A}$ model is described by parameter $W$. This parameter determines both eddy current instantaneous losses, as well as anomalous losses connected with domain-wall motion. Parameter $W$ is given by the following Eqs. [8, 11]:

$$
\begin{aligned}
& W=r_{1} \frac{\mathrm{d} M \mathrm{~d} M}{\mathrm{~d} t \mathrm{~d} H_{\mathrm{eff}}}+r_{2}\left(\frac{\mathrm{d} M}{\mathrm{~d} t}\right)^{1 / 2} \frac{\mathrm{d} M}{\mathrm{~d} H_{\mathrm{eff}}}, \\
& r_{1}=\frac{d^{2} \mu_{0}}{2 \varphi \beta}, \\
& r_{2}=\sqrt{\frac{G d w H_{0} \mu_{0}}{\varphi}},
\end{aligned}
$$

where $G$ is the dimensionless constant equal to $0.1356, w$ is the width and $d$ is the thickness of laminations, $\varphi$ is the resistivity of the material and $H_{0}$ is an equivalent of the magnetic field, describing internal potential experienced by domain walls, whereas $\beta$ is a geometrical factor, describing the shape of the magnetized sample $[8,11]$.

In the case of the family of hysteresis loops, obtained for different values of maximal magnetizing field, the quality of modelling decreases significantly. This phenomenon is caused mainly by the fact that the $\mathrm{J}-\mathrm{A}$ model parameter $k$ changes during the magnetization process [12]. Changes of parameter $k$ are caused by changes of the average energy required to break pinning site [13]. For this reason, the J-A model parameter $k$ can be connected with the magnetic state of the material (described by magnetization $M$ ) by the following equation [14]:

$$
k=k_{0}+\frac{\mathrm{e}^{k_{2}\left(1-|M| / M_{s}\right)}-1}{\mathrm{e}^{k_{2}}-1}\left(k_{1}-k_{0}\right),
$$

where $M_{s}$ is the saturation magnetization, whereas $k_{0}, k_{1}$ and $k_{2}$ describe the shape of the function determining $k$.

It should be indicated that in many papers $[7,10]$ converted form of the $\mathrm{J}-\mathrm{A}$ model equation is used. When the changes of parameter $k$, given by Eq. (6), are considered, the differential J-A model equations have to be solved numerically.

In the $\mathrm{J}-\mathrm{A}$ model, the reversible magnetization $M_{\text {rev }}$ can be calculated from Eq. [6]:

$$
M_{\mathrm{rev}}=c\left(M_{\mathrm{an}}-M_{\mathrm{irr}}\right),
$$

where $c$ describes the magnetization reversibility. Total magnetization $M$ is given as the sum of reversible magnetization $M_{\text {rev }}$ and irreversible magnetization $M_{\text {irr }}$ [6]. As a result, total flux density $B$ in the magnetic core can be calculated as $B=M \mu_{0}+H \mu_{0}$.

Results of the numerical calculations presented in [8] indicate that considering only the eddy current instantaneous losses and anomalous losses (described by Eqs. (3)(5)) is not sufficient for the successful modelling of the frequency dependence of magnetic characteristics, with an adequate accuracy. For this reason, the original $\mathrm{J}-\mathrm{A}$ model should be extended, by considering the frequency dependence of parameters $c$ and $a$. Such extension is physically judged due to the fact that both the magnetization reversibility (described by parameter $c$ ), as well as domain wall density (described by parameter $a$ ) depend on the frequency of the magnetizing field. As a result, such new extension of the J-A model gives better physical description of frequency dependence of magnetization process.

\section{Experimental procedure}

For the extended J-A model its parameters were calculated on the base of the experimental results of measurements of quasi-static magnetization characteristics of $\mathrm{Fe}_{40} \mathrm{Ni}_{38} \mathrm{Mo}_{4} \mathrm{~B}_{18}$ amorphous alloy, in as-quenched state. The core with the height of $10 \mathrm{~mm}$, inner dimension of $25 \mathrm{~mm}$ and outside dimension of $32 \mathrm{~mm}$, was made of an amorphous alloy ribbon with $25 \mu \mathrm{m}$ thickness.

On the base of the experimental $B(H)$ hysteresis loops, values of the extended J-A model parameters were calculated during the optimization process. It should be indicated that during the optimization process, the target function (sum of square of differences between model and experimental results) was calculated simultaneously for 7 hysteresis loops, measured for different value of frequency of magnetizing field, varying from $1 \mathrm{~Hz}$ up to $500 \mathrm{~Hz}$. Moreover, physical aspects of possible values of calculated parameters was also considered to avoid unrealistic conditions, such as negative values of parameters connected with energy or magnetization.

The target function $F$ for the $\mathrm{J}-\mathrm{A}$ model is continuous, but exhibits many local minima. As a result, the evolutional strategies $(\mu+\lambda)$ [15] together with simulated annealing [16] in the first step, and then in the second step the gradient optimization can be applied [14] to find the $\mathrm{J}-\mathrm{A}$ model parameters in physically judged range. Evolutionary strategy $(\mu+\lambda)$ is a time consuming calculation, but exhibits a strongly limited sensitivity to local minima of the target function $F[15]$.

\section{Results}

During the optimization process the following parameters of the J-A model were achieved: $k_{0}=244.93 \mathrm{~A} / \mathrm{m}$, $k_{1}=14.82 \mathrm{~A} / \mathrm{m}, k_{2}=-10.86, M_{s}=3.34 \times 10^{5} \mathrm{~A} / \mathrm{m}$, $\alpha=2.17 \times 10^{4}, K_{\text {an }}=1881 \mathrm{~J} / \mathrm{m}^{3}, t=0.842, r_{1}=$ $4.56 \times 10^{-9}, r_{2}=2.58 \times 10^{-6}$. This means that the average sample thickness $d$, calculated from Eq. (5), equals about $250 \mu \mathrm{m}$, whereas the ribbon thickness was $25 \mu \mathrm{m}$. This differences may be caused by the fact that ribbons in the core were not isolated, but a significant resistance of randomly located contacts between ribbons in the wound core occurred.

The results of the modelling of the frequency dependence of magnetic hysteresis loops of $\mathrm{Fe}_{40} \mathrm{Ni}_{38} \mathrm{Mo}_{4} \mathrm{~B}_{18}$ amorphous alloy in as-quenched state are presented in Fig. 1. It should be underlined that very good agreement between experimental data and the results of the modelling using the extended $\mathrm{J}-\mathrm{A}$ model was observed. 


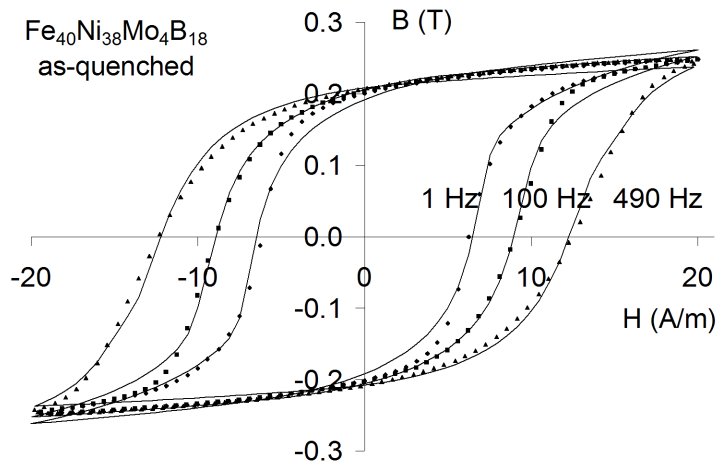

Fig. 1. The results of the modelling of the influence of frequency on $B(H)$ hysteresis loop of amorphous alloy: solid line - results of the modeling, - experimental data.

This very good agreement is confirmed by values of the Pearson $R^{2}$ coefficient, which describes the part of total changes of the measured variable that is described by the model. For all values of the frequency, up to about $500 \mathrm{~Hz}$, coefficient $R^{2}$ exceeds $99.7 \%$.

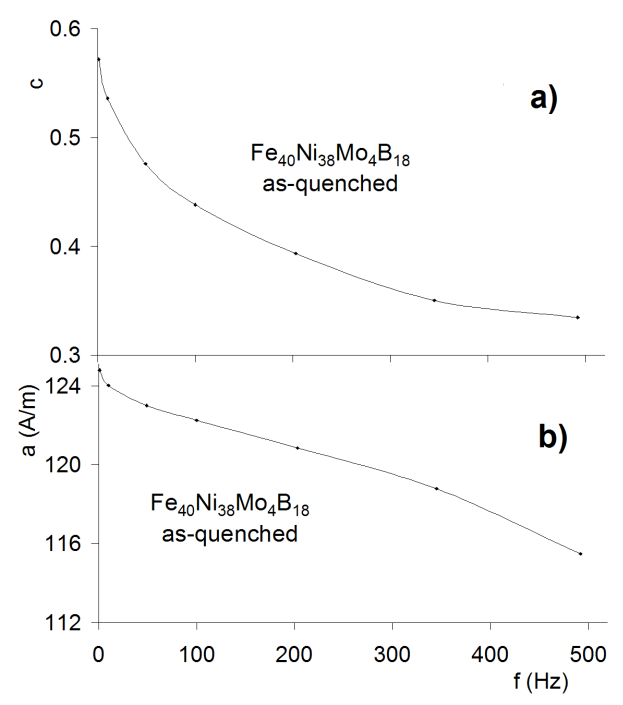

Fig. 2. The influence of the frequency on J-A model parameters: (a) frequency dependence of parameter $c$, (b) frequency dependence of parameter $a$.

In Fig. 2 the frequency dependences of the J-A model parameters are presented. The value of parameter $c$ decreases when frequency increases, due to the fact that the magnetic coupling is weaker for higher values of the magnetizing field frequency. Also, parameter $a$ decreases significantly for higher values of magnetizing field frequency. This phenomenon is probably connected with the changes of domain wall density in the core magnetized with higher frequency. For higher frequency of the magnetizing field the domain wall density increases, which causes decrease of the value of the parameter $a$.

\section{Conclusion}

Presented extension of the Jiles-Atherton model enables modelling of the frequency dependence of magnetic characteristics of $\mathrm{Fe}_{40} \mathrm{Ni}_{38} \mathrm{Mo}_{4} \mathrm{~B}_{18}$ amorphous alloy in as-quenched state, with accuracy sufficient for technical applications in modelling the cores of inductive components for electronic devices. For all hysteresis loops generated with magnetizing field frequency up to about $500 \mathrm{~Hz}$, very good agreement between experimental hysteresis loops and the model occurs. This good agreement is confirmed by the value of the Pearson $R^{2}$ coefficient, which for all hysteresis loops exceeds $99.7 \%$.

For calculation of the J-A model parameters, evolutional strategy $(\mu+\lambda)$ was used. Results of calculation are in the good agreement with physical values describing magnetic core. However, calculated ribbon thickness about $250 \mu \mathrm{m}$ may take into account the fact that ribbons in the core were not effectively isolated.

\section{Acknowledgments}

Calculations for the modelling were made in the Interdisciplinary Centre for Mathematical and Computational Modelling of Warsaw University, within grant G36-10.

\section{References}

[1] F. Liorzou, B. Phelps, D.L. Atherton, IEEE Trans. Magn. 36, 418 (2000).

[2] P. Ripka, Sensors Actuators A 33, 129 (1992).

[3] A. Bieńkowski, R. Szewczyk, Sensors and Actuators $A$ 113, 270 (2004).

[4] A. Pressman, Switching Mode Power Supply Design, McGraw-Hill, New York 1998.

[5] P. Andrei, O. Caltun, A. Stancu, IEEE Trans. Magn. 34, 231 (1998).

[6] D.C. Jiles, D. Atherton, J. Magn. Magn. Mater. 61, 48 (1986).

[7] M.J. Sablik, D.C. Jiles, IEEE Trans. Magn. 35, 498 (1999).

[8] D.C. Jiles, IEEE Trans. Magn. 30, 4326 (1994).

[9] A. Ramesh, D.C. Jiles, J. Roderik, IEEE Trans. Magn. 32, 4234 (1996).

[10] K. Chwastek, J. Szczyglowski, Math. Comp. Simulation 71, 206 (2006).

[11] P. Kis, A. Ivanyi, Physica B 343, 59 (2004).

[12] D.C. Jiles, D.L. Atherton, J. Appl. Phys. 55, 2115 (1984).

[13] J.A. Jatau, E. Della Torre, IEEE Trans. Magn. 30, 4927 (1994).

[14] R. Szewczyk, J. Phys. D 40, 4109 (2007).

[15] H.P. Schwefel, Evolution and Optimum Seeking, Wiley, New York 1995.

[16] H.P. Schwefel, Numerical Optimalization of Numerical Models, Wiley, New York 1981. 